\begin{tabular}{|c|c|c|}
\hline 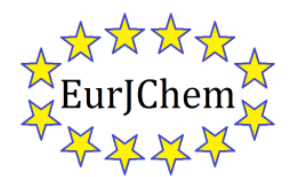 & $\begin{array}{c}\text { European Journal of Chemistry } \\
\text { Journal homepage: } \underline{\text { www.eurjchem.com }}\end{array}$ & 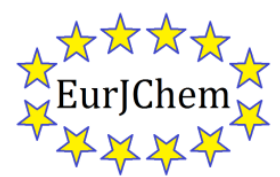 \\
\hline
\end{tabular}

\title{
Synthesis of new 4,6-disubstituted-1,3-5-triazin-2-yloxy esters and $N$-hydroxyamides
}

\author{
Svetlana Mikhaylichenko*, Olga Kvak, Shadi Dalili and Vladimir Zaplishny \\ Department of Physical and Environmental Sciences, University of Toronto Scarborough, Toronto, ON-M1C 1A4, Canada \\ *Corresponding author at: Department of Physical and Environmental Sciences, University of Toronto Scarborough, Toronto, ON-M1C 1A4, Canada. \\ Tel.: +14162877207; fax: +14162877204. E-mail address: mikhay@utsc.utoronto.ca (S. N. Mikhaylichenko).
}

\section{ARTICLE INFORMATION}

\section{Received: 25 June 2010}

Received in revised form: 29 September 2010

Accepted: 07 October 2010

Online: 31 December 2010

\section{KEYWORDS}

\section{3,5-Triazines}

Alkylation

Transesterification

Hydroxamic acids

Synthesis

\begin{abstract}
A convenient method for synthesis of new sym-triazine ester and hydroxamate derivatives has been developed. Various reaction conditions were studied and optimized, and a series of new 1,3,5-triazine based esters and $N$-hydroxyamides were obtained with good yields (38$80 \%)$. The reaction between oxo-derivatives of 4,6-disubstituted-1,3,5-triazines and halogenated carboxylic esters derivatives using $\mathrm{Cs}_{2} \mathrm{CO}_{3}$ as a catalyst was found to be the most convenient method for 4,6-disubstituted-1,3,5-triazine-2-yloxy esters synthesis. These 1,3,5triazin based esters served as precursors for the synthesis of 4,6-disubstituted-1,3,5-triazin2 -yloxy- $N$-hydroxybutanamides using solution of hydroxylamine hydrochloride in dry methanol and $\mathrm{KOH}$ at room temperature. Structures of the newly synthesized compounds were obtained by ${ }^{1} \mathrm{H}$ NMR, ${ }^{13} \mathrm{C}$ NMR, MS, IR spectral data and elemental analysis.
\end{abstract}

\section{Introduction}

Hydroxamic acids (HA) were discovered by Hoffman in 1889 [1] and till this day their high biological activity and practical value [2-6] attract the interest of many researchers. Hydroxamic acids are good chelators and widely used in analytical chemistry [4]. HA can also be used for fishing out heavy and transition metals from diluted solutions of their salts $[7,8]$. However, the biggest attraction to the chemistry of hydroxamic acids is due their high biological activity [9-16] such as inhibitors for different enzymatic systems [9,10], carcenolytics [11-13], killers against plant-parasitic in various organisms [14,15], as well as very active antimalarial compounds [16].

Furthermore, 1,3,5-triazine derivatives posses a wide variety of biological activity as HIV treatment, and as catalysts, herbicides, carcenolytics [17-20], as well as compounds with growth-stimulating activity, antidote activity, nontoxic cationic surfactants, and inhibitors of the oil oxidizing processes [2128].

Combination of these two bioactive moieties in one structure could enhance their existing bioactivities. Synthesis and investigation of some physical properties of new hydroxamic acids containing the sym-triazine backbone (THA) was the subject of our interest in this research.

Hydroxamic acids can be synthesized from amino acids and hydroxyl amine using cyanuric chloride acid as a catalyst [29]. The most common method of HA synthesis is treating the ester derivatives with hydroxyl amine [12,30-35]. Although some sym-triazine containing esters have been described, which were synthesized through a multistep synthesis using melamine and isocyanates [30], only one article was found where the sym-triazine based hydroxamic acids have been described [31]. The initial reagent for the synthesis of these
THA was melamine and these hydroxamic acids have been used for the investigation of the gel formation processes [31].

Both methods $[30,31]$ allow synthesis of only $\alpha$-amino acid ester, amide, or hydroxamate derivatives of sym-triazines. The syntheses of hydroxamates of oxy-acid derivatives of symtriazines have not been previously described.

\section{Experimental}

\subsection{Instrumentation and materials}

The IR spectra were recorded on a BRUKER-Alpha $\mathrm{P}$ spectrophotometer. The ${ }^{13} \mathrm{C}$ and ${ }^{1} \mathrm{H}$ NMR spectra of esters and hydroxamates were measured on a Varian-400 radiospectrometer in $\mathrm{CHCl}_{3}-\mathrm{D}_{1}$ and $\mathrm{DMSO}^{-\mathrm{D}_{6}}$ solution accordingly. The mass-spectra were obtained on a Finnigan MAT INCOS50 instrument (ionizing radiation energy was 70 eV). Elemental analysis was carried out on a Carlo-Erba model 1106 analyzer. The progress of the reactions was monitored and the purities of the compounds were checked by TLC on Polymer-2 with UV sensitive silufol layer plates in a acetone:hexane (1:1) system.

The initial 2,4-disubstituted-6-chloro-1,3,5-triazines, starting ammonium salts (I) and oxocompounds (II) were prepared from cyanuric chloride according to the procedures described earlier $[2,7,36,37]$. The solvents have been purified and dried according to the procedures as described earlier [38, 39].

\subsection{Synthesis}

\subsubsection{Compounds III a-k}

Compounds III a-k were synthesized similarly. Ethyl 4-(4,6dimorpholino-1,3,5-triazine-2-yloxy)butanoate (IIIh): $1.337 \mathrm{~g}$ 
Table 1. IR, NMR and Mass Spectra of new esters and hydroxamic acids-1,3,5-triazine derivatives.

\begin{tabular}{|c|c|c|c|c|}
\hline Compound & IR-spectrum, $v\left(\mathrm{~cm}^{-1}\right)$ & 1H NNR $\delta(p p m)$ & ${ }^{13} \mathrm{C}$ NMR $\delta(p p m)$ & $\begin{array}{c}\text { MS } m / z \\
\left(I_{\text {rel }}, \%\right)\end{array}$ \\
\hline$\overline{\text { IIIa }}$ & $\begin{array}{l}1728(\mathrm{C}=0) ; 1604 ; 1568 ; 1530(- \\
\mathrm{C}=\mathrm{C}+-\mathrm{C}=\mathrm{N}) ; 1263(-\mathrm{C}-\mathrm{N}) ; 1160 ; \\
1123 ; 1084 ; 1008(\mathrm{C}-\mathrm{O}-\mathrm{C})\end{array}$ & 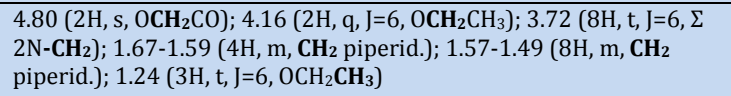 & $\begin{array}{l}\text { 178.4, 174.4, 169.3 } \\
65.5,61.3,52.4 \\
25.9,25.5,14.1\end{array}$ & $349(65)$ \\
\hline IIIb & $\begin{array}{l}1744(\mathrm{C}=0) ; 1582,1539 ; 1496(- \\
\mathrm{C}=\mathrm{C}+-\mathrm{C}=\mathrm{N}) ; 1255(-\mathrm{C}-\mathrm{N}) ; 1155 \\
1107 ; 1063 ; 1001(\mathrm{C}-\mathrm{O}-\mathrm{C})\end{array}$ & $\begin{array}{l}4.74(2 \mathrm{H}, \mathrm{s}, \mathrm{OCH} 2 \mathrm{CO}) ; 4.20\left(2 \mathrm{H}, \mathrm{q}, \mathrm{J}=6, \mathrm{OCH}_{2} \mathrm{CH}_{3}\right) ; 3.79(8 \mathrm{H}, \mathrm{t}, \mathrm{J}=6, \Sigma \\
\left.2 \mathrm{~N}-\mathrm{CH}_{2}\right) ; 3.64\left(8 \mathrm{H}, \mathrm{t}, \mathrm{J}=6, \Sigma \mathrm{OCH}_{2} \mathrm{CH}_{2} \mathrm{~N}\right) ; 1.25\left(3 \mathrm{H}, \mathrm{t}, \mathrm{J}=6, \mathrm{OCH}_{2} \mathbf{C H}_{3}\right)\end{array}$ & $\begin{array}{l}178.8,176.5,169.3 \\
66.4,65.4,62.2 \\
46.3,15.5\end{array}$ & $353(77)$ \\
\hline IIIC & $\begin{array}{l}3081,2976(\mathrm{CH} \mathrm{Ph}) ; 1754(\mathrm{C}=0) \\
\text { 1590, } 1553,1499(-\mathrm{C}=\mathrm{C}+-\mathrm{C}=\mathrm{N}) \\
1195 ; 1134 ; 1069 ; 1020(\mathrm{C}-\mathrm{O}-\mathrm{C})\end{array}$ & $\begin{array}{l}\text { 7.44-7.34, 7.32-7.22, 7.21-7.11 (10H, m, } \Sigma \mathbf{C H ~ O P h}) ; 4.98(2 \mathrm{H}, \mathrm{s}, \\
\left.\mathrm{OCH}_{2} \mathbf{C = 0}\right) ; 4.23\left(2 \mathrm{H}, \mathrm{q}, \mathrm{J}=5.4 ; \mathrm{OCH}_{2} \mathrm{CH}_{3}\right) ; 1.29(3 \mathrm{H}, \mathrm{t}, \mathrm{J}=5.4 ; \\
\left.\mathrm{OCH}_{2} \mathbf{C H}_{3}\right)\end{array}$ & $\begin{array}{l}181,174.6,168.5 \\
155.2,129.8,124.7 \\
121.3,65.6,62.5 \\
18.5\end{array}$ & $367(100)$ \\
\hline IIId & $\begin{array}{l}1578,1530 ; 1498(-\mathrm{C}=\mathrm{C}+-\mathrm{C}=\mathrm{N}) \\
1269(\mathrm{C}-\mathrm{N}) ; 1174,1118,1083 \\
1022(\mathrm{C}-\mathrm{O}-\mathrm{C})\end{array}$ & $\begin{array}{l}4.31\left(2 \mathrm{H}, \mathrm{t}, \mathrm{J}=6.0 ; \mathrm{OCH}_{2}\left(\mathrm{CH}_{2}\right)_{2} \mathrm{CO}\right) ; 4.13\left(2 \mathrm{H}, \mathrm{q}, \mathrm{J}=6 ; \mathrm{OCH}_{2} \mathrm{CH}_{3}\right) ; 3.72 \\
\left(8 \mathrm{H}, \mathrm{t}, \mathrm{\Sigma} 2 \mathbf{C H}_{2} \mathrm{~N}-\mathbf{C H}_{2}\right) ; 2.47\left(2 \mathrm{H}, \mathrm{t}, \mathrm{J}=6.0 ;-\mathbf{C H}_{2} \mathrm{C}=0\right) ; 2.16(2 \mathrm{H}, \mathrm{t} . \mathrm{t} ; \mathrm{J}=6 ; \\
\left.\mathrm{OCH}_{2} \mathbf{C H}_{2} \mathrm{CH}_{2}\right) ; 1.68-1.59\left(4 \mathrm{H}, \mathrm{m}, \mathbf{C H}_{2} \text { piperid. }\right) ; 1.58-1.49(8 \mathrm{H}, \mathrm{m}, \\
\left.\mathbf{C H}_{2} \text { piperid. }\right) ; 1.21\left(3 \mathrm{H}, \mathrm{t}, \mathrm{J}=6, \mathrm{OCH}_{2} \mathbf{C H}_{3}\right)\end{array}$ & $\begin{array}{l}178.4,171.4,122.8 \\
70.4,65.5,49.7 \\
36.1,31.1,30.09 \\
29.9,19.1\end{array}$ & $377(65)$ \\
\hline IIIe & $\begin{array}{l}1744(\mathrm{C}=0) ; 1587,1538,1504(- \\
\mathrm{C}=\mathrm{C}+-\mathrm{C}=\mathrm{N}) ; 1165,1122,1089 \\
1017(\mathrm{C}-\mathrm{O}-\mathrm{C})\end{array}$ & $\begin{array}{l}4.43\left(2 \mathrm{H}, \mathrm{t}, \mathrm{J}=6.3 ; \mathbf{C H}_{2}\left(\mathrm{CH}_{2}\right)_{2} \mathrm{CO}\right) ; 4.11\left(2 \mathrm{H}, \mathrm{q}, \mathrm{J}=6.3 ; \mathrm{OCH}_{2} \mathrm{CH}_{3}\right) ; 3.91 \\
\left(6 \mathrm{H}, \mathrm{s}, \mathrm{OCH}_{3}\right) ; 2.53\left(2 \mathrm{H}, \mathrm{t}, \mathrm{J}=6.3 ;-\mathbf{C H}_{2} \mathrm{C}=0\right) ; 2.21(2 \mathrm{H}, \mathrm{t} . \mathrm{t} . ; \mathrm{J}=6.3 ; \\
\left.\mathrm{OCH}_{2} \mathbf{C H}_{2} \mathrm{CH}_{2}\right) ; 1.23\left(3 \mathrm{H}, \mathrm{t}, \mathrm{J}=4.8 ; \mathrm{OCH}_{2} \mathbf{C H}_{3}\right)\end{array}$ & $\begin{array}{l}177.9,175.7,170.7 \\
70.03,65.3,45.8 \\
36.1,19.4\end{array}$ & $71(60)$ \\
\hline IIIf & $\begin{array}{l}1732(\mathrm{C}=0) ; 1580,1528,1501(- \\
\mathrm{C}=\mathrm{C}+-\mathrm{C}=\mathrm{N}) ; 1252(-\mathrm{C}-\mathrm{N}) ; 1180 \\
1109,1049,1006(\mathrm{C}-\mathrm{O}-\mathrm{C})\end{array}$ & $\begin{array}{l}4.40\left(2 \mathrm{H}, \mathrm{t}, \mathrm{J}=5.4 ; \mathrm{OCH}_{2}\left(\mathrm{CH}_{2}\right)_{2}\right) ; 4.14\left(2 \mathrm{H}, \mathrm{q}, \mathrm{J}=5.4 ; \mathrm{OCH}_{2} \mathrm{CH}_{3}\right) ; 3.94 \\
(3 \mathrm{H}, \mathrm{s}, \mathrm{OCH}) ; 3.86\left(4 \mathrm{H}, \mathrm{t}, \mathrm{J}=5.4 ; \Sigma \mathrm{NCH}_{2}\right) ; 3.71\left(4 \mathrm{H}, \mathrm{t}, \mathrm{J}=5.4 ; \mathrm{OCH}_{2}\right) ; \\
2.50\left(2 \mathrm{H}, \mathrm{t}, \mathrm{J}=5.4 ; \mathbf{C H}_{2} \mathrm{CO}\right) ; 2.15-2.06\left(2 \mathrm{H}, \mathrm{m}, \mathrm{COCH}_{2} \mathbf{C H}_{2}\right) ; 1.25(3 \mathrm{H}, \mathrm{t}, \\
\left.\mathrm{J}=5.4 ; \mathrm{OCH}_{2} \mathbf{C H}_{3}\right)\end{array}$ & $\begin{array}{l}182,173.5,173.1 \\
169.8,68.3,66.3 \\
61.2,55.8,46.5 \\
30.1,24.5,19.2\end{array}$ & $326(68)$ \\
\hline IIIg & $\begin{array}{l}3062,2961(\mathrm{CH} \mathrm{Ph}) ; 1727(\mathrm{C}=0) \\
1598,1562,1497(\mathrm{C}=\mathrm{C}+\mathrm{C}=\mathrm{N}) \\
1163,1125,1091,1021(\mathrm{C}-\mathrm{O}-\mathrm{C})\end{array}$ & $\begin{array}{l}\text { 7.45-7.35, 7.28-7.19, 7.18-7.10 (10H, m, } \Sigma \text { CHOPh }) ; 4.33(2 \mathrm{H}, \mathrm{t}, \\
\left.\mathrm{J}=6.0 ; \mathrm{OCH}_{2}\left(\mathrm{CH}_{2}\right)_{2} \mathrm{CO}\right) ; 4.12\left(2 \mathrm{H}, \mathrm{q}, \mathrm{J}=6.0 ; \mathrm{OCH}_{2} \mathrm{CH}_{3}\right) ; 2.53(2 \mathrm{H}, \mathrm{t}, \\
\left.\mathrm{J}=6.0 ;-\mathbf{C H}_{2} \mathrm{CO}\right) ; 2.09-2.00\left(2 \mathrm{H}, \mathrm{m}, \mathrm{OCH}_{2} \mathbf{C H}_{2} \mathrm{CH}_{2}\right) ; 1.30(3 \mathrm{H}, \mathrm{t}, \mathrm{J}=6.0 ; \\
\left.\mathrm{OCH}_{2} \mathbf{C H}_{3}\right)\end{array}$ & $\begin{array}{l}179.1,178.1,157.5 \\
135.1,131.6,127.1 \\
122.8,73.2,65.7 \\
35.7,29.2,19.1\end{array}$ & $395(86)$ \\
\hline IIIh & $\begin{array}{l}1728(\mathrm{C}=0) ; 1574,1531,1498 \\
(\mathrm{C}=\mathrm{C}+\mathrm{C}=\mathrm{N}) ; 1251(-\mathrm{C}-\mathrm{N}) ; 1183 \\
1115,1047,1021(\mathrm{C}-\mathrm{O}-\mathrm{C})\end{array}$ & $\begin{array}{l}4.32\left(2 \mathrm{H}, \mathrm{t}, \mathrm{J}=6.0 ; \mathrm{OCH}_{2} \mathrm{CH}_{2} \mathrm{CH}_{2}\right) ; 4.14\left(2 \mathrm{H}, \mathrm{q}, \mathrm{J}=6.0 ; \mathrm{OCH}_{2} \mathrm{CH}_{3}\right) ; 3.80 \\
(8 \mathrm{H}, \mathrm{t}, \mathrm{J}=6.0 ; \Sigma \mathrm{OCH}) ; 3.68\left(8 \mathrm{H}, \mathrm{t}, \mathrm{J}=6.0 ; \mathrm{NCH}_{2} \mathrm{CH}_{2} \mathrm{O}\right) ; 2.46(2 \mathrm{H}, \mathrm{t}, \\
\left.\mathrm{J}=6.0 ; \mathbf{C H}_{2} \mathrm{CO}\right) ; 2.15-2.06\left(2 \mathrm{H}, \mathrm{m}, \mathrm{CH}_{2} \mathbf{C H}_{2} \mathrm{CH}_{2} \mathrm{CO}\right) ; 1.23(3 \mathrm{H}, \mathrm{t}, \mathrm{J}=6.0 ; \\
\left.\mathrm{OCH}_{2} \mathbf{C H}_{3}\right)\end{array}$ & $\begin{array}{l}178.5,175.4,172.9 \\
68.4,66.9,61.6 \\
47.1,30.3,24.4,18.9\end{array}$ & $381(57)$ \\
\hline IIIi & $\begin{array}{l}1736(\mathrm{C}=0) ; 1591,1523,1499(- \\
\mathrm{C}=\mathrm{C}+\mathrm{C}=\mathrm{N}) ; 1284(\mathrm{CN}) ; 1174 \\
1129,1105,1030(\mathrm{C}-\mathrm{O}-\mathrm{C})\end{array}$ & $\begin{array}{l}4.35\left(2 \mathrm{H}, \mathrm{t}, \mathrm{J}=5.5 ; \mathrm{OCH}_{2}\left(\mathrm{CH}_{2}\right)_{2}\right) ; 4.11\left(2 \mathrm{H}, \mathrm{q}, \mathrm{J}=5.5 ; \mathrm{OCH}_{2} \mathrm{CH}_{3}\right) ; 3.96 \\
(3 \mathrm{H}, \mathrm{s}, \mathrm{OCH} 3) ; 3.79-3.71\left(4 \mathrm{H}, \mathrm{m}, \Sigma \mathrm{NCH}_{2}\right) ; 2.46\left(2 \mathrm{H}, \mathrm{t}, \mathrm{J}=5.5 ; \mathrm{COCH}_{2}\right) ; \\
2.12-2.03\left(2 \mathrm{H}, \mathrm{m}, \mathrm{OCH}_{2} \mathbf{C H}_{2} \mathrm{CH}_{2}\right) ; 1.68-1.61\left(2 \mathrm{H}, \mathrm{m}, \mathbf{C H}_{2} \text { piperid. }\right) \\
1.59-1.52\left(4 \mathrm{H}, \mathrm{m}, \mathbf{C H}_{2} \text { piperid. }\right) ; 1.23\left(3 \mathrm{H}, \mathrm{t}, \mathrm{J}=5.5 ; \mathrm{OCH}_{2} \mathbf{C H}_{3}\right)\end{array}$ & $\begin{array}{l}181.7,173.3,173.1 \\
169.5,68.5,62.5 \\
56.1,52.4,30.7 \\
25.9,25.5,24.6,20.4\end{array}$ & $324(71)$ \\
\hline IIIj & $\begin{array}{l}1731(\mathrm{C}=0) ; 1574,1529,1498 \\
(\mathrm{C}=\mathrm{C}+\mathrm{C}=\mathrm{N}) ; 1256(\mathrm{CN}) ; 1158 \\
1111,1095,1034(\mathrm{C}-\mathrm{O}-\mathrm{C})\end{array}$ & $\begin{array}{l}4.32\left(2 \mathrm{H}, \mathrm{t}, \mathrm{J}=5.8 ; \mathrm{OCH}_{2} \mathrm{CH}_{2}\right) ; 4.12\left(2 \mathrm{H}, \mathrm{q}, \mathrm{J}=5.8 ; \mathrm{OCH}_{2} \mathrm{CH}_{3}\right) ; 3.95(3 \mathrm{H}, \\
\mathrm{s}, \mathrm{OCH} 3) ; 3.84\left(4 \mathrm{H}, \mathrm{t}, \mathrm{J}=5.8 \Sigma \mathrm{NCH}_{2}\right) ; 3.72\left(4 \mathrm{H}, \mathrm{t}, \mathrm{J}=5.8 ; \Sigma \mathrm{OCH}_{2}\right) ; 2.32 \\
\left(2 \mathrm{H}, \mathrm{t}, \mathrm{J}=5.8 ; \mathbf{C H}_{2} \mathrm{CO}\right) ; 1.85-1.76\left[2 \mathrm{H}, \mathrm{m}, \mathrm{OCH}_{2} \mathbf{C H}_{2}\left(\mathrm{CH}_{2}\right)_{3}\right] ; 1.73-1.64 \\
\left(2 \mathrm{H}, \mathrm{m}, \mathrm{O}\left(\mathrm{CH}_{2}\right)_{3}-\mathrm{CH}_{2}\right) ; 1.53-1.44\left(2 \mathrm{H}, \mathrm{m}, \mathrm{O}\left(\mathrm{CH}_{2}\right)_{2} \mathbf{C H}_{2}\left(\mathrm{CH}_{2}\right)_{2} \mathrm{CO}\right) ; \\
1.26\left(3 \mathrm{H}, \mathrm{t}, \mathrm{J}=5.4 ; \mathrm{OCH}_{2} \mathbf{C H}_{3}\right)\end{array}$ & $\begin{array}{l}178.8,176.5,171.8 \\
122.8,71.8,71.5 \\
49.1,39.2,35.4 \\
33.8,30.7,29.9,19.1\end{array}$ & $354(60)$ \\
\hline IIIk & $\begin{array}{l}3063,2978(\mathrm{CHPh}) ; 1730(\mathrm{C}=0) \\
1596,1553,1499(-\mathrm{C}=\mathrm{C}+-\mathrm{C}=\mathrm{N}) \\
1162,1115,1081,1029(\mathrm{C}-\mathrm{O}-\mathrm{C})\end{array}$ & $\begin{array}{l}\text { 7.44-7.34, 7.28-7.18, 6.96-6.86 (10H, m, } \Sigma \mathbf{C H O P h}) ; 4.13(2 \mathrm{H}, \mathrm{q}, \\
\left.\mathrm{J}=6.0 ; \mathrm{OCH}_{2} \mathrm{CH}_{3}\right) ; 3.95\left(2 \mathrm{H}, \mathrm{t}, \mathrm{J}=6.0 ; \mathbf{O C H}_{2} \mathrm{CH}_{2}\right) ; 2.36(2 \mathrm{H}, \mathrm{t}, \mathrm{J}=6.0 ; \\
\left.\mathbf{C H}_{2} \mathrm{CO}\right) ; 1.84-1.75\left(2 \mathrm{H}, \mathrm{m}, \mathrm{OCH}_{2} \mathbf{C H}_{2}\left(\mathrm{CH}_{2}\right)_{3} \mathrm{CO}\right) ; 1.59-1.50(2 \mathrm{H}, \mathrm{m}, \mathrm{O} \\
\left.\left(\mathrm{CH}_{2}\right)_{3} \mathbf{C H}_{2} \mathrm{CH}_{2} \mathrm{CO}\right) ; 1.43-1.34\left(2 \mathrm{H}, \mathrm{m}, \mathrm{OCH}_{2} \mathrm{CH}_{2} \mathbf{C H}_{2} \mathrm{CH}_{2} \mathrm{CH}_{2} \mathrm{CO}\right) ; 1.27 \\
\left(3 \mathrm{H}, \mathrm{t}, \mathrm{J}=6.0 ; \mathrm{OCH}_{2} \mathbf{C H}_{3}\right)\end{array}$ & $\begin{array}{l}181.7,173.3,173.1 \\
169.5,68.9,66.4 \\
62.5,55.8,46.9 \\
33.9,29.5,25.9,20.4\end{array}$ & $423(45)$ \\
\hline IVa & $\begin{array}{l}3204(\mathrm{O}-\mathrm{H}, \mathrm{N}-\mathrm{H}) \\
1672(\mathrm{C}=\mathrm{Oamid}) ; 1576,1515 \\
1499(\mathrm{C}=\mathrm{C}+\mathrm{C}=\mathrm{N}) ; 1251(\mathrm{C}-\mathrm{N}) \\
1202,1114,1067,1004(\mathrm{C}-\mathrm{O}-\mathrm{C})\end{array}$ & $\begin{array}{l}8.22(1 \mathrm{H}, \mathrm{s}, \mathrm{HO}-\mathrm{NH}) ; 4.75\left(2 \mathrm{H}, \mathrm{s}, \mathrm{OCH}_{2} \mathrm{CO}\right) ; 3.77(8 \mathrm{H}, \mathrm{t}, \mathrm{J}=3.0 ; \\
\left.\Sigma \mathrm{NCH}_{2} \mathrm{CH}_{2} \mathrm{O}\right) ; 3.68\left(8 \mathrm{H}, \mathrm{t}, \mathrm{J}=3.0 ; \Sigma \mathrm{OCH}_{2} \mathrm{CH}_{2} \mathrm{~N}\right) ; 1.78(1 \mathrm{H}, \mathrm{s}, \mathrm{HO}-\mathrm{NH})\end{array}$ & $\begin{array}{l}170.1,168.2,166.1 \\
66.6,44.1,40.6\end{array}$ & $340(38)$ \\
\hline IVb & $\begin{array}{l}3258(\mathrm{O}-\mathrm{H}, \mathrm{N}-\mathrm{H}) ; 1693 \\
(\mathrm{C}=\text { Oamid }) ; 1567,1528,1497 \\
(\mathrm{C}=\mathrm{C}+\mathrm{C}=\mathrm{N}) ; 1250(\mathrm{CN}) ; 1163 \\
1107,1047,1008(\mathrm{C}-\mathrm{O}-\mathrm{C})\end{array}$ & $\begin{array}{l}8.36(1 \mathrm{H}, \mathrm{s}, \mathrm{HO}-\mathrm{NH}) ; 4.34\left(2 \mathrm{H}, \mathrm{t}, \mathrm{J}=4.0 ; \mathrm{OCH}_{2}\left(\mathrm{CH}_{2}\right)_{2} \mathrm{CO}\right) ; 3.78(8 \mathrm{H}, \mathrm{t}, \\
\left.\mathrm{J}=4.0 ; \Sigma \mathrm{NCH}_{2}\right) ; 3.70\left(8 \mathrm{H}, \mathrm{t}, \mathrm{J}=4.0 ; \Sigma \mathrm{OCH}_{2}\right) ; 2.46\left(2 \mathrm{H}, \mathrm{t}, \mathrm{J}=4.0 ; \mathbf{C H}_{2} \mathrm{CO}\right) ; \\
2.18(1 \mathrm{H}, \mathrm{s}, \mathbf{H O}-\mathrm{NH}) ; 2.13-2.04\left(2 \mathrm{H}, \mathrm{m}, \mathrm{OCH}_{2} \mathbf{C H}_{2} \mathrm{CH}_{2}\right)\end{array}$ & $\begin{array}{l}170.9,169.2,165.9 \\
67.9,66.4,44.3 \\
31.4,24.9\end{array}$ & $368(53)$ \\
\hline IVc & $\begin{array}{l}3274(\mathrm{O}-\mathrm{H}, \mathrm{N}-\mathrm{H}) ; 1688 \\
(\mathrm{C}=\mathrm{Oamid}) ; 1577,1532,1498 \\
(\mathrm{C}=\mathrm{C}+\mathrm{C}=\mathrm{N}) ; 1239(\mathrm{CN}) ; 1154 \\
1122,1049,1006(\mathrm{C}-\mathrm{O}-\mathrm{C})\end{array}$ & $\begin{array}{l}8.43(1 \mathrm{H}, \mathrm{s}, \mathrm{HO}-\mathrm{NH}) ; 4.34\left(2 \mathrm{H}, \mathrm{t}, \mathrm{J}=4.8 ; \mathrm{OCH}_{2}\left(\mathrm{CH}_{2}\right)_{2} \mathrm{CO}\right) ; 3.97(3 \mathrm{H}, \mathrm{s}, \\
\left.\mathrm{OCH}_{3}\right) ; 3.71\left(8 \mathrm{H}, \mathrm{t}, \mathrm{J}=4.8 ; \Sigma \mathrm{N}-\mathrm{CH}_{2} \mathrm{CH}_{2} \mathrm{O}\right) ; 3.71(8 \mathrm{H}, \mathrm{t}, \mathrm{J}=4.8 ; \\
\left.\Sigma \mathrm{OCH}_{2} \mathrm{CH}_{2} \mathrm{~N}\right) ; 2.49\left(2 \mathrm{H}, \mathrm{t}, \mathrm{J}=4.8 ;-\mathbf{C H}_{2} \mathrm{CO}\right) ; 2.48(1 \mathrm{H}, \mathrm{s}, \mathrm{HO}-\mathrm{NH}) ; 2.13- \\
2.04\left(2 \mathrm{H}, \mathrm{m}, \mathrm{OCH}_{2} \mathbf{C H}_{2} \mathrm{CH}_{2}\right)\end{array}$ & $\begin{array}{l}182,173.1,169.9 \\
67.9,66.4,55.8 \\
46.4,28.8,25.0\end{array}$ & $327(100)$ \\
\hline IVd & $\begin{array}{l}3041,2811(\mathrm{CH} P h) ; 1696 \\
(\mathrm{C}=\text { Oamid }) ; 1570,1548,1498 \\
(\mathrm{C}=\mathrm{C}+\mathrm{C}=\mathrm{N}) ; 1169,1115,1060 \\
1010(\mathrm{C}-\mathrm{O}-\mathrm{C})\end{array}$ & $\begin{array}{l}8.42(1 \mathrm{H}, \mathrm{s}, \mathrm{HO}-\mathrm{NH}) ; 7.57-7.47,7.38-7.28,7.22-7.12(10 \mathrm{H}, \mathrm{m}, \\
\Sigma \mathbf{C H O P h}) ; 4.33\left(2 \mathrm{H}, \mathrm{t}, \mathrm{J}=5.1 ; \mathrm{OCH}_{2} \mathrm{CH}_{2} \mathrm{CH}_{2} \mathrm{CO}\right) ; 2.53(2 \mathrm{H}, \mathrm{t}, \mathrm{J}=5.1 ; \\
\left.\mathbf{C H}_{2} \mathrm{CO}\right) ; 2.29(1 \mathrm{H}, \mathrm{s}, \mathrm{HO}-\mathrm{NH}) ; 2.08-2.00\left(2 \mathrm{H}, \mathrm{m}, \mathrm{OCH}_{2} \mathbf{C H}_{2} \mathrm{CH}_{2}\right)\end{array}$ & $\begin{array}{l}181,174.5,169.8 \\
155.2,129.8,124.8 \\
121.3,68.2,27.9 \\
24.8\end{array}$ & $410(48)$ \\
\hline IVe & $\begin{array}{l}3239(\mathrm{O}-\mathrm{H}, \mathrm{N}-\mathrm{H}) ; 1703 \\
(\mathrm{C}=\text { Oamid }) ; 1594,1562 ; 1496 \\
(\mathrm{C}=\mathrm{C}+-\mathrm{C}=\mathrm{N}) ; 1239(\mathrm{CN}) ; 1186 \\
1118,1047,1011(\mathrm{C}-\mathrm{O}-\mathrm{C})\end{array}$ & $\begin{array}{l}8.51(1 \mathrm{H}, \mathrm{s}, \mathrm{HO}-\mathrm{NH}) ; 4.11\left(2 \mathrm{H}, \mathrm{t}, \mathrm{J}=6.0 ; \mathbf{O C H}_{2} \mathrm{CH}_{2} \mathrm{CH}_{2} \mathrm{CO}\right) ; 3.16(8 \mathrm{H}, \mathrm{t}, \\
\left.\Sigma \mathbf{C H}_{2} \mathrm{~N}\right) ; 2.48(1 \mathrm{H}, \mathrm{s}, \mathbf{H O}-\mathrm{NH}) ; 2.27\left(2 \mathrm{H}, \mathrm{t}, \mathrm{J}=6.0 ;-\mathbf{C H}_{2} \mathrm{C}=0\right) ; 2.14-2.06 \\
\left(2 \mathrm{H}, \mathrm{m}, \mathrm{OCH}_{2} \mathbf{C H}_{2} \mathrm{CH}_{2}\right) ; 1.58-1.49\left(4 \mathrm{H}, \mathrm{m}, \mathbf{C H}_{2} \text { pypired. }\right) ; 1.51-1.42 \\
\left(8 \mathrm{H}, \mathrm{m}, \mathbf{C H}_{2} \text { pypired.) }\right.\end{array}$ & $\begin{array}{l}178.6,173.5,170.2 \\
67.7,52.4,28.8 \\
26.1,25.5,25.0\end{array}$ & $364(78)$ \\
\hline IVf & $\begin{array}{l}3220(\mathrm{O}-\mathrm{H}, \mathrm{N}-\mathrm{H}) ; 1684 \\
(\mathrm{C}=\text { Oamid); } 1561,1531,1498 \\
(\mathrm{C}=\mathrm{C}+\mathrm{C}=\mathrm{N}) ; 1247(\mathrm{C}-\mathrm{N}) ; 1193 \\
1150,1106,1002(\mathrm{C}-\mathrm{O}-\mathrm{C})\end{array}$ & $\begin{array}{l}8.12(1 \mathrm{H}, \mathrm{s}, \mathrm{HO}-\mathrm{NH}) ; 4.27\left(2 \mathrm{H}, \mathrm{t}, \mathrm{J}=6.3 ; \mathrm{OCH}_{2} \mathrm{CH}_{2}\right) ; 3.55(8 \mathrm{H}, \mathrm{t}, \mathrm{J}=6.3 ; \\
\left.\Sigma \mathrm{NCH}_{2}\right) ; 3.46\left(8 \mathrm{H}, \mathrm{t}, \mathrm{J}=6.3 ; \Sigma \mathrm{OCH}_{2}\right) ; 2.40\left(2 \mathrm{H}, \mathrm{t}, \mathrm{J}=6.3 ; \mathrm{CH}_{2} \mathrm{CO}\right) ; 2.09 \\
(1 \mathrm{H}, \mathrm{s}, \mathrm{HO}-\mathrm{NH}) ; 1.83-1.75\left(2 \mathrm{H}, \mathrm{m}, \mathrm{OCH}_{2} \mathbf{C H}_{2}\left(\mathrm{CH}_{2}\right)_{3} \mathrm{CO}\right) ; 1.74-1.65 \\
\left(2 \mathrm{H}, \mathrm{m}, \mathrm{O}\left(\mathrm{CH}_{2}\right)_{3} \mathrm{CH}_{2} \mathrm{CH}_{2} \mathrm{CO}\right) ; 1.53-1.44\left(2 \mathrm{H}, \mathrm{m}, \mathrm{OCH}_{2} \mathrm{CH}_{2-}\right. \\
\left.\mathbf{C H}_{2} \mathrm{CH}_{2} \mathrm{CH}_{2} \mathrm{CO}\right)\end{array}$ & $\begin{array}{l}173.9,173.5,171.0 \\
66.6,66.3,40.6 \\
33.8,29,25.7,25.0\end{array}$ & $396(61)$ \\
\hline
\end{tabular}


Table 2. Characteristics of Synthesized compounds IIIa-k, IVa-f, Va.

\begin{tabular}{|c|c|c|c|c|c|c|}
\hline \multirow{2}{*}{ Compound } & \multirow{2}{*}{ Empirical formula } & \multicolumn{3}{|c|}{ Found (Calculated) (\%) } & \multirow{2}{*}{ M.p. $\left({ }^{\circ} \mathrm{C}\right)$} & \multirow{2}{*}{ Yield (\%) } \\
\hline & & $\mathbf{C}$ & $\mathbf{H}$ & $\mathbf{N}$ & & \\
\hline IIIa & $\mathrm{C}_{17} \mathrm{H}_{27} \mathrm{~N}_{5} \mathrm{O}_{3}$ & $58.23(58.43)$ & $8.02(7.79)$ & $20.29(20.04)$ & $84-86$ & 52.7 \\
\hline IIIb & $\mathrm{C}_{15} \mathrm{H}_{23} \mathrm{~N}_{5} \mathrm{O}_{5}$ & $51.17(50.98)$ & $6.41(6.56)$ & $19.66(19.82)$ & $90-92$ & 73.0 \\
\hline IIIC & $\mathrm{C}_{19} \mathrm{H}_{17} \mathrm{~N}_{3} \mathrm{O}_{5}$ & $62.25(62.12)$ & $4.52(4.66)$ & $11.60(11.44)$ & $75-78$ & 47.3 \\
\hline IIId & $\mathrm{C}_{19} \mathrm{H}_{31} \mathrm{~N}_{5} \mathrm{O}_{3}$ & $60.58(60.45)$ & $8.41(8.28)$ & $18.63(18.55)$ & $60-62$ & 68.0 \\
\hline IIIe & $\mathrm{C}_{11} \mathrm{H}_{17} \mathrm{~N}_{3} \mathrm{O}_{5}$ & $48.53(48.70)$ & $6.47(6.32)$ & $15.67(15.49)$ & $76-78$ & 48.4 \\
\hline IIIf & $\mathrm{C}_{14} \mathrm{H}_{22} \mathrm{~N}_{4} \mathrm{O}_{5}$ & $55.37(55.52)$ & $6.58(6.79)$ & $17.29(17.17)$ & $40-42$ & 59.7 \\
\hline IIIg & $\mathrm{C}_{21} \mathrm{H}_{21} \mathrm{~N}_{3} \mathrm{O}_{5}$ & $63.64(63.79)$ & $5.51(5.35)$ & $10.52(10.63)$ & $70-72$ & 50.8 \\
\hline IIIh & $\mathrm{C}_{17} \mathrm{H}_{27} \mathrm{~N}_{5} \mathrm{O}_{5}$ & $53.45(53.53)$ & $4.28(4.14)$ & $18.49(18.36)$ & $73-75$ & 69.2 \\
\hline IIII & $\mathrm{C}_{15} \mathrm{H}_{24} \mathrm{~N}_{4} \mathrm{O}_{4}$ & $55.66(55.54)$ & $7.58(7.45)$ & $17.39(17.27)$ & $38-40$ & 40.1 \\
\hline IIIj & $\mathrm{C}_{16} \mathrm{H}_{24} \mathrm{~N}_{4} \mathrm{O}_{5}$ & $54.36(54.22)$ & 7.19 (7.39) & $15.95(15.81)$ & $34-36$ & 41.2 \\
\hline IIIk & $\mathrm{C}_{23} \mathrm{H}_{25} \mathrm{~N}_{3} \mathrm{O}_{5}$ & $65.38(65.21)$ & $5.84(5.95)$ & $9.83(9.92)$ & $62-65$ & 49.5 \\
\hline IVa & $\mathrm{C}_{13} \mathrm{H}_{20} \mathrm{~N}_{6} \mathrm{O}_{5}$ & $45.69(45.88)$ & $5.78(5.92)$ & $24.76(24.69)$ & $146-148$ & 47.9 \\
\hline IVb & $\mathrm{C}_{15} \mathrm{H}_{24} \mathrm{~N}_{6} \mathrm{O}_{5}$ & $48.45(48.90)$ & $6.28(6.57)$ & $22.98(22.81)$ & $158-160$ & 54.1 \\
\hline IVc & $\mathrm{C}_{13} \mathrm{H}_{21} \mathrm{~N}_{5} \mathrm{O}_{5}$ & $47.76(47.64)$ & $6.58(6.49)$ & $21.19(21.40)$ & $150-153$ & 41.5 \\
\hline IVd & $\mathrm{C}_{20} \mathrm{H}_{20} \mathrm{~N}_{4} \mathrm{O}_{5}$ & $58.41(58.53)$ & $5.04(4.91)$ & $17.21(17.06)$ & $140-141$ & 46.3 \\
\hline IVe & $\mathrm{C}_{17} \mathrm{H}_{28} \mathrm{~N}_{6} \mathrm{O}_{3}$ & $56.18(56.03)$ & $7.84(7.74)$ & $23.18(23.06)$ & $179-180$ & 38.4 \\
\hline IVf & $\mathrm{C}_{17} \mathrm{H}_{28} \mathrm{~N}_{6} \mathrm{O}_{5}$ & $51.36(51.50)$ & $7.29(7.12)$ & $21.35(21.20)$ & $182-183$ & 65.9 \\
\hline Va & $\mathrm{C}_{16} \mathrm{H}_{25} \mathrm{~N}_{5} \mathrm{O}_{5}$ & $52.03(52.25)$ & $6.97(6.80)$ & $19.36(19.05)$ & 101-103 & 72.1 \\
\hline
\end{tabular}

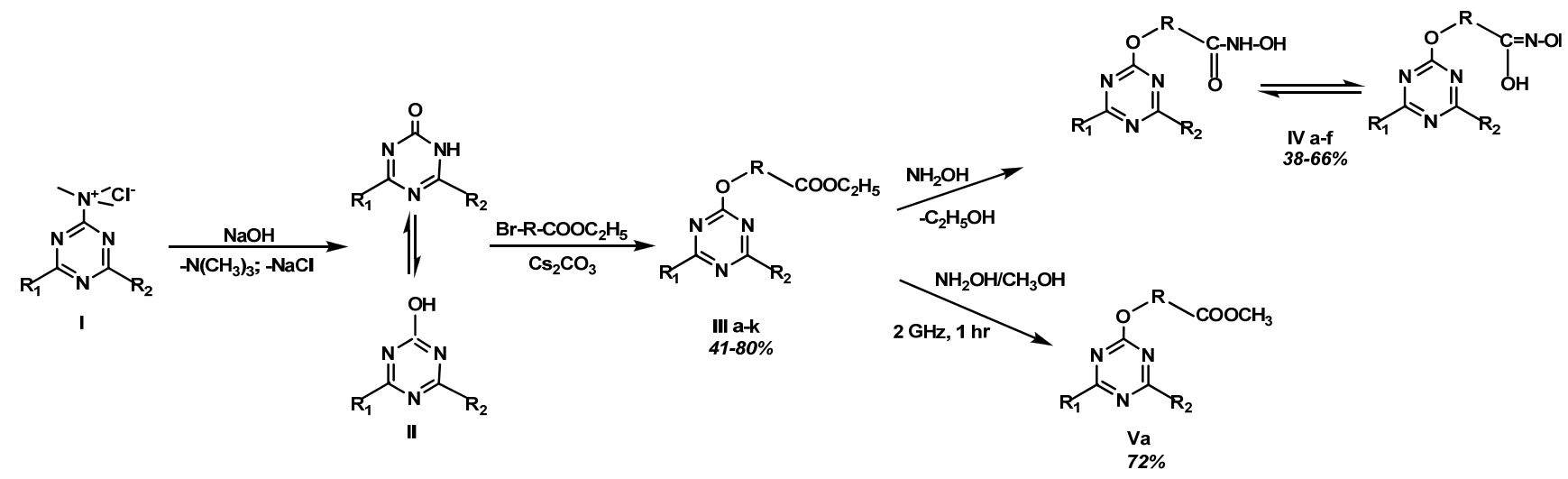

IIIa,d R $\mathrm{R}_{1}=\mathrm{R}_{2}=$ Piperidino, $\mathrm{R}=\mathrm{CH}_{2}$ IIIa, $\left(\mathrm{CH}_{2}\right)_{3}$ IIId; IIIb,h Va R $\mathrm{R}_{1}=\mathrm{R}_{2}=$ Morpholino, $\mathrm{R}=\mathrm{CH}_{2}$ IIIb, Va; $\left(\mathrm{CH}_{2}\right)_{3}$ IIIh, IIIf,j $\mathrm{R}_{1}=\mathrm{OCH}_{3}, \mathrm{R}_{2}=\mathrm{Morpholino}_{1} \mathrm{R}=(\mathrm{CH})_{3}$ IIIf, $\left(\mathrm{CH}_{2}\right)_{5}$ IIIj; IIIc,g,k $\mathrm{R}_{1}=\mathrm{R}_{2}=\mathrm{OC}_{6} \mathrm{H}_{5}, \mathrm{R}=\mathrm{CH}_{2}$ IIIc, $\left(\mathrm{CH}_{2}\right)_{3}$ IIIg, $\left(\mathrm{CH}_{2}\right)_{5}$ IIIk; IIIe $\mathrm{R}_{1}=\mathrm{R}_{2}=\mathrm{OCH}_{3}, \mathrm{R}=\left(\mathrm{CH}_{2}\right)_{3} ;$ IIIi $\mathrm{R}_{1}=\mathrm{OCH}_{3}, \mathrm{R}_{2}=\mathrm{Piperidino} \mathrm{R}=\left(\mathrm{CH}_{2}\right)_{3} ;$ IVa,b,f $\mathrm{R}_{1}=\mathrm{R}_{2}=$ Morpholino, $\mathrm{R}=\mathrm{CH}_{2}$ IVa, $\left(\mathrm{CH}_{2}\right)_{3}$ IVb, $\left(\mathrm{CH}_{2}\right)_{5}$ IVf; IVc $\mathrm{R}_{1}=\mathrm{OCH}_{3}, \mathrm{R}_{2}=$ Morpholino, $\mathrm{R}=\left(\mathrm{CH}_{2}\right)_{3} ; \mathbf{I V d} \mathrm{R}_{1}=\mathrm{R}_{2}=\mathrm{OC}_{6} \mathrm{H}_{5}, \mathrm{R}=\left(\mathrm{CH}_{2}\right)_{3} ; \mathbf{I V e} \mathrm{R}_{1}=\mathrm{R}_{2}=\mathrm{Piperidino}, \mathrm{R}=\left(\mathrm{CH}_{2}\right)_{3}$.

Figure 1. Synthesis of the (2,4-disubstituted-1,3,5-triazin-2-yloxy)- $N$-hydroxyamides and esters.

(4.99 mmol) of 4,6-dimorpholino-1,3,5-triazin-2(1H)-one was dissolved in $15 \mathrm{~mL}$ of dry $\mathrm{DMF}$ at $60{ }^{\circ} \mathrm{C}$ and stirred for $10 \mathrm{~min}$ and then the solution of $1.114 \mathrm{~g}(4.99 \mathrm{mmol})$ ethyl 4-bromobutanoate and $1.735 \mathrm{~g}(5.49 \mathrm{mmol}) \mathrm{Cs}_{2} \mathrm{CO}_{3}$ in $15 \mathrm{~mL}$ DMF was added drop wise. The reaction mixture was stirred at $60-80{ }^{\circ} \mathrm{C}$ for 4 hours. Progress of the reaction was monitored by TLC. The solution was allowed to cool down to $20^{\circ} \mathrm{C}$ and $25 \mathrm{~mL}$ of ice cold water was added with continued stirring. The reaction mixture was kept on an ice bath approximately 2-3 hour to ensure that maximum amount of white precipitate was obtained. The product was filtered and washed out with cold water $(3 \times 20 \mathrm{~mL})$ and dried at $50{ }^{\circ} \mathrm{C}$. After purification by crystallization from ethanol:water solution (3:2), $1.316 \mathrm{~g}$ of compound IIIh was obtained (69.2\%) (Figure 1). Physicochemical characteristics of synthesized compounds could be found in Table 1 and 2 .

\subsubsection{Compounds IV a, $c-f$}

Compounds IV a, c-f were prepared similarly. 4-(4,6Dimorpholino-1,3,5-triazin-2-yloxy)- $N$-hydroxybutanamide (IVb): A solution of $0.639 \mathrm{~g}(9.2 \mathrm{mmol})$ hydroxylamine hydrochloride in $10 \mathrm{~mL}$ of dry methanol and $0.515 \mathrm{~g}(9.2$ mmol) $\mathrm{KOH}$ in $10 \mathrm{~mL}$ dry methanol were mixed at room temperature. The reaction mixture was stirred in an ice bath for $10 \mathrm{~min}$ and filtered. The resulting solution was slowly added to a solution of $1.16 \mathrm{~g}(3.045 \mathrm{mmol})$ of ethyl 4-(4,6dimorpholino-1,3,5-triazine-2-yloxy)butanoate in $25 \mathrm{~mL}$ of dry methanol. The reaction mixture was refluxed for 6 hours and progress of the reaction was monitored by TLC. The solution was allowed to cool down to $20^{\circ} \mathrm{C}$. The reaction mixture was evaporated by vacuum. The resulting residue was dried at $50{ }^{\circ} \mathrm{C}$ and purified by crystallization from iso-propanol:water (3:1) solution (Figure 1). The final yield was $0.606 \mathrm{~g}$ (54.1\%). Physico-chemical characteristics of synthesized compounds could be found in Table 1 and 2 .

\subsubsection{Compounds Va}

Methyl 4-(4,6-dimorpholino-1,3,5-triazine-2-yloxy)butanoate (Va): A solution of $0.639 \mathrm{~g}(9.2 \mathrm{mmol})$ hydroxylamine hydrochloride in $10 \mathrm{~mL}$ of dry methanol and $0.515 \mathrm{~g}(9.2$ $\mathrm{mmol}$ ) $\mathrm{KOH}$ in $10 \mathrm{~mL}$ dry methanol were mixed at room temperature. The reaction mixture was stirred in an ice bath for $10 \mathrm{~min}$ and filtered. The resulting solution was slowly added to a solution of $1.16 \mathrm{~g}(3.045 \mathrm{mmol})$ of ethyl 4-(4,6dimorpholino-1,3,5-triazine-2-yloxy)butanoate in $25 \mathrm{~mL}$ of dry methanol. The reaction mixture was heated in microwave at $2 \mathrm{GHz}$ for 1 hour and progress of the reaction was monitored by TLC. The solvent was evaporated by vacuum. The product was filtered and washed out with cold water $(3 \times 20 \mathrm{~mL})$ and dried at $50{ }^{\circ} \mathrm{C}$. After purification by crystallization from ethanol: water solution (3:2), 0.83 g of compound Va was obtained $(72 \%)$ (Figure 1). Physico-chemical characteristics of synthesized comp-ound could be found in Table 1 and 2 .

\section{Results and discussion}

We investigated several different methods to synthesize the desired THA as shown in Figure 1. The attempts to synthesize 
hydroxamates from sym-triazine carboxylic acids were not successful even using cyanuric chloride as a catalyst. This method is also not very suitable due to the limited number of hydroxyl containing acids available. It was possible to synthesize THA using esters III as intermediate compounds. This could be achieved using 4,6 disubstituted-2(trimethylamino) chlorides I as initial compounds [36,37]. Unfortunately, the attempts to synthesize esters III with direct reaction between quaternary salts $\mathbf{I}$ and some esters of oxycarboxylic acids were not successful probably due to the relatively low nucleophilicity of the hydroxy group in these acids. Salts hydrolysis products (II) were the major products in all of these cases. We found that alkylation of oxo-derivatives II is the most convenient and successful method for the esters (III) synthesis. Compounds II could be easily synthesized by the basic hydrolysis of quaternary salts [36].

The progress of the reactions was monitored using TLC. It is interesting to note that the alkylation of the oxo-derivatives II with the $\omega$-bromo-alkenylesters in the presence of equimolar amounts of $\mathrm{KOH}, \mathrm{NaOH}$, or $\mathrm{K}_{2} \mathrm{CO}_{3}$ as acceptors of $\mathrm{HBr}$ takes about several days to complete with very low yields (10-15\%). Increasing the reaction time, as well as using different types of purified protic solvents [38,39] did not affect the final product yields. Alternatively, using dry DMF as a solvent and equimolar amount of $\mathrm{Cs}_{2} \mathrm{CO}_{3}$ as a base have been found to be optimal conditions for this synthesis. This helped to increase the final yields to $39-80 \%$ (see Table 1) as well as decrease the reaction time from few days to several hours.

The sym-triazine ester derivatives (III) were treated with three equivalents of freshly prepared hydroxyl amine in dry methanol to produce the final products [12,32-35]. The final yields under these conditions were $38-66 \%$. Attempts to increase the final product's yield using KCN [34] as a catalyst or microwave conditions [12] were not very successful. The main product of reaction between compound IIIh and hydroxylamine using dry methanol as a solvent under microwave synthesis conditions was transesterefication product Va. Using THF as a solvent for microwave synthesis did not produce the desired product with a reasonable yield.

The new compounds III and IV are white or light yellow crystals. They are not soluble in water and alkanes; THA have a good solubility only in dioxane, DMF, DMSO, and other organic solvents with a high boiling point; esters are soluble in alcohols, dichloromethane, and chloroform. Compounds III have lower melting points $\left(34-95^{\circ} \mathrm{C}\right)$ compared with compounds IV (140$182{ }^{\circ} \mathrm{C}$ ), as expected, due to the absence of the hydrogen bonds. The melting point values for both types of sym-triazine derivatives were lower with longer carbon chain length.

The structures of all synthesized compounds established by IR, ${ }^{13} \mathrm{C}$ NMR, ${ }^{1} \mathrm{H}$ NMR, Mass-spectroscopy, and Elemental analysis data (see Tables 1,2 ).

IR-spectroscopy peaks for compounds III and IV show variable intensity, with broad absorption bands as well as stretching vibrations typical for the functional groups in their structures. Compounds III and IV have three medium and strong absorption bands in the area 1603-1496 $\mathrm{cm}^{-1}$ typical for the conjugated $\mathrm{C}=\mathrm{C}$ and $\mathrm{C}=\mathrm{N}$. The $\mathrm{C}=0$ singlet absorption band is seen for compounds III present at $1729-1760 \mathrm{~cm}^{-1}$, while this signal has been shifted to higher frequencies $\left(1703-1672 \mathrm{~cm}^{-1}\right)$ in case of THA. This is typical for the $\mathrm{C}=\mathrm{O}_{\text {amid. }}$. Compounds III and IV all have four absorption bands at 1195-1002 $\mathrm{cm}^{-1}$ typical for the C-O-C functional group. IR spectra of compounds IIIc, IIIg and IIIk have medium and small absorption bands at 815-806 and 787-773 $\mathrm{cm}^{-1}$ typical for the bending vibrations of the $\gamma_{\mathrm{CH}}$ in $\mathrm{Ph}$.

${ }^{1} \mathrm{H}$ NMR spectra data of all synthesized compounds have signals of all corresponding protons and the integration curves prove the number of protons. The single proton of the $\mathrm{NH}$ amide has a signal in the area of 8.12-8.51 ppm. Compounds IV have the singlet signal of the $\mathrm{OH}-\mathrm{NH}$ group at $1.78-2.48 \mathrm{ppm}$, which is the most prominent structural distinction between these compounds. The protons of the morpholyl and piperidyl rings of derivatives III and IV appear as multiplet signals (see Table 1).

The mass spectroscopy spectra of compounds III and IV also confirm their structure. Compounds IIIc and IVc have the maximum intensity of the molecular ions. The fragmentation pattern of the molecular ions under electronic impact is the same as has been observed for the synthesized heterocyclic derivatives of the 1,3,5-triazine [36,37,40-43].

In summary, a series of new organic compounds containing ester and hydroxamate derivatives of 1,3,5-triazine have been prepared. The alkylation of sym-triazine oxo-derivatives was found to be the most convenient and successful method for the oxy-acid esters with triazine backbone. Treatment of these esters with freshly prepared hydroxylamine in methanol successfully lead to the new hydroxamate derivatives of 1,3,5triazine. The investigation of the practical bioactivity of these derivatives is the subject for our future research.

\section{Acknowledgement}

We would like to thank the Chairman of the Physical and Environmental Department of UTSC Professor Donald E. Cormack without whose support this research would be impossible to complete.

\section{References}

[1]. Knuniants I. Chem. Encyclopedia, 5th edition, v. 1, Sovietskaya encyclopedia Publishing House, Moscow, 1988.

[2]. Fazary, A. E.; Khalil, M. M.; Fahmy, A.; Tantawy, T. A. Medical Journal of Islamic Academy of Sciences, 2001, 14(3), 109-116.

[3]. Dooley, C. M.; Devocelle, M.; McLoughlin, K. B.; Nolan, K. B.; Fitzgerald D. J.; Sharkey, C. T. Mol. Pharmacol. 2003, 63(2), 450-455.

[4]. Nigović, B.; Kujundžić, N.; Sanković, K.; Vikić-Topić, D. Acta Chim. Slov. 2002, 49(3), 525-535.

[5]. Nirmal, K.; Huber, V.; Smith, P.; Gopalan, A. Tetrahedron, 1994, 50(9), 2657-2664.

[6]. Huang, L.; Pardee, A. B. Mol. Medicine, 2000, 6(10), 849-866.

[7]. Taylor, R. Czech. J. Phys. 1999, 49, 617-623.

[8]. Matijevic, J.; Samarzija, I.; Honovic, L.; Jurisic, B. Acta Pharm. 2008, 58, 231-236.

[9]. Kobashi, K.; Takebe, S.; Terashima, N.; Hase, J. J. Biochem. 1975, 77, 837-843.

[10]. Mishra, H.; Parrill, A. L.; Williamson, J. S. Antimicrob. Agents Chemother. 2002, 46(8), 2613-2618.

[11]. Emanuele, S.; Lauricella, M.; Tesoriere, G. Int. J. Oncol. 2008, 33(4), 637-646.

[12]. Massaro, A.; Mordini, A.; Reginato, G.; Russo, F.; Taddei M. Synthesis 2007, 20, 3201-3204

[13]. Ning, L.; Greenblatt, D. Y.; Kunnimalaiyaan, M.; Chen, H. Oncologist, 2008, 13(2), 98-104.

[14]. Niemeyer, H. M. Euphytica, 1988, 37(3), 289-293.

[15]. Zasada, I.; Meer, S.; Halbrendt, J.; Rice C. Nematology, 2005, 95, 11161121.

[16]. Holland, K. P.; Elford, H. L.; Bracchi, V.; Annis, C. G.; Schuster, S. M.; Chakrabarti, D. Antimicrob. Agents Chemother. 1998, 42(9), 24562458.

[17]. Blotny, G. Tetrahedron, 2006, 62(41), 9507-9522.

[18]. Smolin, E.; Rappoport, L. s-Triazine and Derivatives, Interscience, NY, 1959.

[19]. Melnikov, N.; Bascakov, Y. Chemistry of Herbicides and Growth Regulators of Plants, Goskhimizdat Publishing House, Moscow, 1962.

[20]. Pogosian, G.; Pankratov, V.; Zaplishny, V.; Matsoyan, S.; Korshak, V. Polytriazines, Armenian SSR Academy of Sciences Publishing House, Erevan, 1987.

[21]. Katritzky, A.; Rees, C.; Scriven, E.; Potts, K. Comprehensive Heterocyclic Chemistry, v. 5, Pergamon, Oxford, 1984

[22]. Demirayak, S.; Karaburun, A. C.; Beis R. Eur. J. Med. Chem. 2004 39(12), 1089-1095.

[23]. Antonian, S.; Zaplishny, V.; Pogosian, G.; Libinzon, R.; Lavretskaya, E.; Pijov, V.; Bessudnova, N.; Sarkisian, D.; Vatolkina O. KhimikoFarmatsevticheskii Zhurnal 1986, 20(2), 172-175.

[24]. Mikhaylichenko, S.; Dounin, V.; Zaplishny, V. Effectivity of the PASS program in Predicting Biological Activity for sym-Triazine Derivatives. Fourth International Symposium on Computational 
Methods in Toxicology and Pharmacology Integrating Internet Resources, Moscow, Russia, September 1-5, 2007.

[25]. Mikhaylichenko, S.; Dounin, V.; Zaplishny, V.; Chesnyuk, A. Synthesis and Potential Bioactivity of New Pyrazolyl-sym-Triazine Derivatives. Fourth International Symposium on Computational Methods in Toxicology and Pharmacology Integrating Internet Resources, Moscow, Russia, September 1-5, 2007.

[26]. Chesnyuk, A.; Mikhaylichenko, S.; Konyushkin, L.; Kotlyarov, N.; Zaplishny, V. Patent RU, Chem. Abstr. 2005, 144, 22947.

[27]. Chesnyuk, A.; Mikhaylichenko, S.; Firgang, S.; Kotlyarov, N.; Zaplishny, V. Patent RU, Chem. Abstr. 2006, 144, 345097.

[28]. Chesnyuk, A.; Mikhaylichenko, S.; Konyushkin, L.; Kotlyarov, N.; Zaplishny, V. Patent RU, Chem. Abstr. 2005, 144, 345098.

[29]. Giacomelli, G.; Porcheddu , A.; Salaris, M. Org. Lett. 2003, 5(15), 27152717.

[30]. Bethel, W. J.; Rowayton, R. Patent US 4, 939, 213, Chem. Abstr. 1990.

[31]. Maffezzoni, R.; Zanda, M. Tetrahedron Let. 2008, 49(35), 5129-5132.

[32]. Anandan, S. K.; Ward, J. S.; Brokx, R. D.; Denny, T.; Bray, M. R.; Patel, D. V.; Xiao, X. Y. Bioorg. Med. Chem. Lett. 2007, 17(21), 5995-5999.

[33]. Hauser, C. R. Renfrow, W. B. Org. Synth. 1939, 19, 15-17.

[34]. Ho, C. Y.; Strobel, E.; Ralbovsky, J.; Galermo, R. A. J. Org. Chem. 2005, $70(12), 4873-4875$.

[35]. Jones, L. W. and Hurd, C. D. J. Am. Chem. Soc. 1921, 43(11), 2422-2448.

[36]. Chesnyuk, A.; Mikhaylichenko, S.; Zavodnov, V.; Zaplishny, V. Chem. Heterocycl. Compd. 2002, 38(2), 177-182.

[37]. Mikhaylichenko, S.; Chesnyuk, A.; Zavodnik, V.; Firgang, S.; Koniushkin L.; Zaplishny, V. Chem. Heterocycl. Compd. 2002, 38(3), 292-299.

[38]. Gordon, A. J.; Ford, R. A. The Chemist's Companion. Handbook of Practical Data, Techniques, and References, John Wiley\&Sons, 1973.

[39]. Jerry, R. M; Christina, N. H.; Paul, F. S. Techniques in Organic Chemistry, W. H. Freeman, 2006.

[40]. Mikhaylichenko, S.; Chesnyuk, A.; Konyushkin, L.; Zaplishny, V. Russ. Chem. Bull. 2003, 52(10), 2157-2160.

[41]. Chesnyuk, A.; Mikhaylichenko, S.; Konyushkin, L.; Firgang, S.; Zaplishny, V. Russ. Chem. Bull. 2005, 54(8), 1900-1906.

[42]. Mikhaylichenko, S.; Chesnyuk, A.; Konyushkin, L.; Zaplishny, V. Chem. Heterocycl. Compd. 2006, 42(5), 642-647.

[43]. Chesnyuk, A.; Mikhaylichenko, S.; Zaplishny, V.; Konyushkin, L.; Firgang, S.; Chem. Heterocycl. Compd. 2008, 44(3), 339-348.

[44]. Vogel, A. J.; Tatchell, A. R.; Furnis, B. S.; Hannaford, A. J.; Smith, P. W. G. Vogel's Textbook of Practical Organic Chemistry, 5th edition, Prentice Hall, 1996. 